\title{
What Happened to our Document in the Shared Workspace? The Need for Groupware Conventions
}

\author{
Gloria Mark and Wolfgang Prinz \\ GMD-FIT, German National Research Center for Information Technology \\ Schloß Birlinghoven \\ 53754 Sankt Augustin, Germany \\ E-mail: \{Gloria.Mark, Wolfgang.Prinz\}@gmd.de
}

\begin{abstract}
Conventions for conducting work with groupware are essential. They include rules for how the groupware functionality should be used for communication about work, for how data should be processed, and for decision-making. Based on user experiences with PoLITeam, a large groupware system implemented in a German ministry, we report how conventions are difficult to establish and to follow. We illustrate how conventions are necessary in practice, through a cooperative task using a shared workspace. Two solutions are presented to aid users in following conventions.
\end{abstract}

\section{KEYWORDS Groupware, CSCW, conventions, awareness, shared workspace}

\section{INTRODUCTION}

A shared workspace in a groupware system has no idea about managerial work. For that matter, it has no idea about secretarial work. Nor does it have any idea about how people should work on shared documents: how the files should be edited, where the documents should be stored, or which data files should be retained. Moreover, a groupware system cannot predict the actions that users take, nor the effects that they will have on others using the system. A groupware system is like a Tabula Rasa; the system provides the functionality, and the users define their procedures with it. However, the users must coordinate their procedures so that the work can be conducted smoothly.

Although user studies in CSCW have addressed the problem of how groups coordinate their work (e.g. Hughes et al., 1992; Posner and Baecker, 1992) only a few studies have addressed how groupware features can aid coordination. Some examples include Quilt, which offers users a choice of styles to coordinate editing
(Leland, et al., 1988). In the Colab project, the need for a shared reference was realized as an important design consideration (Tatar et al., 1991). PREP is a collaborative editor which employs role assignment and explicit annotation to aid coordination (Neuwirth et al., 1990). However, groupware functionality is becoming increasingly complex, and as users in PoliTeam, a large groupware project, pointed out, coordination problems exist not only with their work, but also in coordinating the use of the functionality itself. In this paper we will discuss how the user requirements have led us to develop a technical solution for how group conventions can be supported, and which allows personal conventions for some work.

\subsection{The PoliTeam project}

The goal of the PoLITeam groupware system is to build a "telecooperation bridge" between Bonn and the new German capital of Berlin. Federal agencies will relocate over time, resulting in a large division of labor 
in departments spread between the old and new capitals. The main function of the PoliTeam system is to supplement paper work processes with electronic work processes in a government ministry. To accomplish this, PoLITeam offers a shared workspace and electronic circulation folders (Prinz and Kolvenbach, 1996). An already existing groupware system (LinkWorks) was chosen and adapted to specific user and situation requirements. For further information, see Klöckner et al. (1995).

Our strategy was to implement prototypes in three representative areas of the government, which enables us to understand how users are adapting, and to correct mistakes early (Orlikowski, 1992). Further, an evolutionary cycling approach was used in the design, allowing modifications to be made over time and which designers and users reported as beneficial (Mambrey et. al, 1996). The project began in May 1994, and the system has been installed since January, 1995. A new version of the system was installed in February, 1996.

The focus of obtaining our user requirements was done with users at the Federal Ministry of Family Affairs, Senior Citizens, Women, and Youth, located in Bonn. In the department where the system is installed, we find varied employee roles: 1 unit leader, 6 ministry employees (responsible for specific content areas of the ministry), and 3 typists in their own service unit. The employees collaborate using the shared workspace and email. Their activities include services such as responding to citizens' requests, doing tasks for the Minister, and collaborative speechwriting. In addition, the manager is responsible for activities such as information dissemination and decision-making.

\section{CONVENTION REQUIREMENTS}

\subsection{Method}

The requirements for user conventions emerged over the course of several workshops. In the most recent workshop, held in June, 1996, the new system version was discussed. The unit leader spoke as representative of the users and stated that the number one user requirement at this time was the need to establish conventions among the users. An in-depth discussion ensued, where all of the users named conventions necessary for their system use, and reasons for them. The PoliTeam design team, along with the users, then discussed the points one by one trying to find solutions to enable the users to set conventions. We did not conduct a formal analysis of this conversation, since we felt that the users presented a straightforward request, and had spent a long time themselves considering how conventions could be set. We attribute this straightforward request to the regular relations established between the users and design team via direct designer-user interaction, and user advocates, which resulted in a long list of other user requirements which were introduced (Mambrey et al., 1996). The earlier discussions of conventions in previous workshops could also have set precedents for the topic.

\subsection{What are conventions?}

We define conventions as rules established in the group, common to all members, for carrying out the following transactions via a groupware system, in particular PoliTeam: communication transactions, e.g. who to inform about document changes; data processing transactions, e.g. should a document be created and distributed as a share or as a copy; and decision-making transactions, e.g. who is responsible for a document, or for maintaining the order of a workspace?

\subsection{Conventions in a shared workspace}

In this section we illustrate how the use of shared workspaces raised the need for the agreement on and support of conventions. With POLITeam, shared folders and email support communication and cooperation. The shared workspace supports a document production task between two units in the ministry: the writing office and a ministry unit (referred to as Unit 57, not its real name). The task of the writing office is to type a draft provided by members of Unit 57.

The shared workspace has two purposes: First, it provides shared access to documents for the writing office and the Unit 57 members for the following: to exchange a text initially produced by the writing office, and to have access to a text for the production of a clear copy that has been produced or that will be modified later by a Unit 57 member. Second, it provides access to all documents that have been produced within the unit to the unit leader.

In the case when the electronic version of the document is modified and then forwarded on paper for 
further processing, the writing office must incorporate the annotated paper changes into the electronic version. Therefore, the writing office requires retaining access to the latest electronic version of the document.

The solution in practice is as follows. Documents are exchanged by all using shared workspaces. For each member of Unit 57 a workspace exists which is shared between this member and members of the writing office. All these workspaces are contained in another folder, called the unit-folder. This allows the writing office to organize documents according to the units and members of a unit. Whenever a document is produced by the writing office, this document is placed in the appropriate unit/person workspace and the person is informed about the new document by an event notification. This convention for how the shared workspaces are organized is logical for the writing office members' work process. It is determined by the name of the user (Unit 57 member) for whom it was typed and the date of production. We refer to this as the order view. The sorting criteria for the documents in a workspace is the date when the document was typed.

This solution seemed appropriate for the users initially. But after some time of practical experience, the following problem occurred. The Unit 57 members wanted to organize their documents according to their work processes, i.e. they wanted to collect documents produced by the writing office in a task or processspecific folder, rather than according to unit members. Their sorting criteria for documents in a workspace depends not on the date of production, but rather on the content of a document, e.g. a speech on a transit problem, or on an economic issue. We refer to this as the work-process view. With the current configuration only one view, the order view, is supported.

Thus, we found that users prefer to structure their information using their own pattern. We also found that the different views of the users corresponded to different level structures: the typists prefer to use a relatively flat information structure, and for some of the unit leaders, they prefer a deep multi-level structure. The problem for the group arises when the different users collaborate in a shared workspace which requires one common information structure for the groups' documents. Some users often could not find documents among the vast array of information in the shared workspace because the system supported the order view, which was not their view.
A second example of a convention is one that was set via a workshop: work must occur in the shared folder. i.e. a document must not be removed. However, in practice, the Unit 57 member would drag the document out of the folder shared with the writing office into their task-specific folder. After that operation, the writing office no longer had access to the electronic document, which was crucial for changes. Thus, the Unit 57 member violated the conventions that were established for the use of the shared folder. Even documents which are produced only by the Unit 57 members must be stored in the folders shared with the writing office, to provide an electronic version for the clear copy production.

\subsection{The user requirements}

The above scenario illustrated how establishing common views of the information, and how agreements about working on shared documents are necessary conventions in a shared workspace. Additionally other conventions that users named in the workshop were:

- Naming conventions for documents. Different users prefer to store and access documents using different naming conventions. The typists preferred naming a document by the creator (or owner) whereas the ministry employees preferred naming documents according to content and semantics.

- Storing old and current documents. The users' one file storage cabinet has become too huge to manage and since more information can be stored electronically, "old" and "new" documents need to be defined.

- Conventions regarding shared tasks. These include what changes to accept in a document; who should make the changes; a model for access rights for different classes of documents; different "closets" (based on an office metaphor, used in POLITeam): working on a document as a share, vs. a copy; editing text; ownership of documents; and conventions for producing new documents.

- Conventions for determining borders between public and private work, in a shared workspace. These also include access rights and document storage.

- Substitution rules for when a workspace member is absent. In the paper world, one can simply lock drawers with personal items, but with groupware, it is not so straightforward, e.g. reading another person's email. 
Conceptual vs. physical owner of a document. One person may be the coordinator of the document, yet another person may have created the document.

Although we present a long list of user requirements, in this paper we focus on presenting general solutions and concepts for the provision of conventions and views in a shared workspace environment.

\section{CONVENTIONS IN THE COURSE OF GROUP DEVELOPMENT}

\subsection{The stages of group development}

To help us design solutions for supporting groupware conventions, we can draw upon behavioral studies to see how groups establish common routines. Although group members bring their own prior experience into a group, a new group starts out without its own culture. Only as members begin to interact does the group develop common goals, means, working procedures, and rules of interaction (Schein, 1985). This process has been modeled extensively as a series group gradually establishes common procedures and goals.

\subsection{The User Group's Evolution with PoLITeam}

We have observed that since the introduction of the PoliTeam system, our user work group has also evolved in its use of the system. We can also characterize the development of our user work group, with respect to its system use, in terms of rough stages. What we have identified over time are changes in attitudes and use of the system. It is important to note that the time span of stages can only be roughly determined, since we could only interview users and hold workshops at specific time points. We believe that although there was probably concurrence with some events, most likely the stages are sequential, i.e. users could not have asked for group conventions without first learning system basics (Table 1).

With respect to the user group in Table 1, we found two interesting phenomena concerning conventions. First, it took about six months of regular use with the

\begin{tabular}{|c|c|c|}
\hline Stage & Time Span & Characteristics of stage: major problems/events \\
\hline $\begin{array}{l}\text { I. Learning basic } \\
\text { functionality; mostly } \\
\text { single-user idea of } \\
\text { system }\end{array}$ & $\begin{array}{l}1 \text { st } 6 \text { months after system } \\
\text { introduced }\end{array}$ & $\begin{array}{l}\text { Problems with windows, hardware, basic computer skills: } \\
\text { - } \quad \text { struggling with text processing } \\
\text { - } \quad \text { transferring individual work practices to system } \\
\text { - } \quad \text { adapting group functionality to meet the group's needs }\end{array}$ \\
\hline $\begin{array}{l}\text { II. Discovering } \\
\text { alternatives for } \\
\text { structuring information }\end{array}$ & between 6 - 9 months & $\begin{array}{l}\text { Developed own style for structuring information: } \\
\text { - } \quad \text { structuring information according to own work process } \\
\text { - } \quad \text { collecting information (finding semantic connections) }\end{array}$ \\
\hline $\begin{array}{l}\text { IV. Mature group } \\
\text { working with the system }\end{array}$ & $?$ & $\begin{array}{l}\text { We would expect the following: } \\
\text { - new, unanticipated use of tools } \\
\text { - conventions would be learned } \\
\text { - implicit conventions would be developed }\end{array}$ \\
\hline
\end{tabular}

Table 1. Stages of user group development with POLITeam

of stages that a group evolves through until such common ground is reached (e.g. Bales, 1950; Bion, 1961; Bennis and Shepard, 1956). Members gather information about the group and use a reciprocal process of accommodation of a group toward the individual, while the individuals attempt to get the group to conform to their style (Moreland and Levine, 1989). This accommodation process continues as the system for the users to discover that they needed conventions for using the system (Stage II in Table 1). Initially, these first conventions that users recognized concerned individual information structuring. It is our view that the users first needed to gain familiarity with the system before it became clear to them that conventions were needed and this required time. When the users became more experienced with the possibilities of the system, they developed ideas for a 
better task and process-oriented support. This resulted in a task-oriented organization and structuring scheme for some users, which conflicts with an initial textprocessing oriented configuration of the system.

It also took time and experience for the users to realize that they needed conventions in order to interact as a group with the system (Stage III). User advocates of PoLITeam made regular site visits to the users' worksites and their support included not only training in system use but also with conventions, such as how the system could support their work practices (Mambrey et. al, 1996). However, it was only with regular use of the system with their unique tasks that made it clear to the users (and to us) that it is not always possible to predict in advance what conventions a group needs in order to use a system like POLITeam. This suggests to us that the process of establishing conventions is on-going and whatever feedback the system can provide about others' activities could benefit this process.

\section{ESTABLISHING CONVENTIONS}

\subsection{The difficulty of setting conventions}

In this section, we discuss why conventions might be difficult to establish with a groupware system, based on our observations during site visits, reports from the user advocates, and conversations with the users during workshops.

1. Restricted feedback and communication makes it difficult to establish conventions. In a face to face group, communication, visual information, and feedback sources are rich, which facilitates the negotiation and agreement of work routines. In contrast, working in distributed locations with groupware restricts social and visual information. In a distributed group, the restricted communication makes it not only more difficult to explicitly set conventions, but also more difficult for people to understand them.

2. The groupware environment lacks cues for conventions. Cues such as type of dress, posture, and tone of speaking in a face-to-face meeting immediately provide information on whether the group is formal or informal, what type of language is appropriate, and whether the group might be following strict rules of procedure or not. A groupware environment lacks such cues, making it difficult for people to know what is appropriate behavior to use with the system.

3. Individuals may be reluctant to give up their individual conventions. Initially PoLITeam users spent a great deal of effort in developing their own styles for sorting information (see Table 1). It may be difficult to give up a style that is meaningful and possibly logical for one's own task. Even in the paper world, people are often reluctant to give up personal conventions.

4. Some conventions with groupware are totally new for the group. With some conventions, users had no analogy from the paper world to apply, such as not knowing when they should distribute documents as 'shares' (a Linkworks feature) or as copies, since the share facility has no counterpart in the paper world. Another example is that a transcriber of a document becomes the owner in a groupware system, and must learn to change the access rights for the actual owner.

5. Unfamiliarity with the system. As outlined in Table 1, users may be unfamiliar with the system, making it difficult for them to understand what conventions might be necessary. System support may also focus too much on teaching functionality, and not enough on coordinating use of the functionality.

\subsection{Means for Supporting Conventions}

Derived from these observations, we consider now methods for how conventions can be established.

In PoLITeam, workshops were conducted to set conventions using user advocates as mediators (Mambrey, 1996). These workshops covered conventions for maintaining public information documents, for document naming conventions, and general concerns. Whereas communication is generally restricted during groupware use, a workshop offers the advantage of face-to-face communication. Users can freely discuss conventions that are needed, along with their goals and purposes. Whereas a groupware environment lacks cues for appropriate behavior, especially for new users, a workshop can help users orient themselves to the groupware context. Setting conventions via workshops can help to overcome the first two difficulties mentioned in section 4.1.

\section{TECHNOLOGICAL SUPPORT}

Technological support can be applied to target the remaining difficulties identified in 4.1. It can facilitate 
convention use in three ways. First, support can be given in the form of awareness. Second, for many routines, default conventions can be implemented into the system. And third, support can enable users to retain individual conventions.

\subsection{Awareness and convention use}

A groupware system, such as a shared workspace, is a social environment. Here, people's actions are not independent. For example, one may make changes to a shared document, which are visible to all others. Not only do people's actions interact with one another, but one's actions can have adverse consequences for others. If a document is removed from a shared workspace, then others may not find it. If someone rearranges files within a folder, creating subdirectories, then others will not readily find them. If someone renames a document, then another user will have serious difficulties.

Many groupware systems, such as a multi-user database, act like single user systems; one can see their own actions, but may be unaware of the actions and their effects from other users. However, a shared workspace is an example of a groupware system where each one's actions and their effects are experienced by those who are cooperating.

In a synchronous shared workspace environment, the effects of users' actions can be immediately seen. For example, Dourish and Bellotti (1992) describe the shared feedback which is available in ShrEdit, a multiuser text editor, where users can see the views of others, or "find" other users in the shared document. Additionally, one has access to activity information; users can see whether others are tracking them or others. This provides information on other users' actions, but not their effects. However, the authors noted that the users would ask each other about effects, such as who had written certain parts, or they would caution each other about consequences of actions.

The immediacy with which actions can be seen in a synchronous environment lends itself to easier learning of the relationship between action and effect, since actions and effects are contiguous in time (e.g. Moeller, 1954). However, asynchronous shared workspaces inhibits learning the relationships of actions-effects, since the time separation makes it more difficult for people to make this connection. One experiences many actions and many effects and along with a time difference, pairing them together can be difficult.
It is for this reason that we argue for awareness information in three time periods. One may see the effects of others, e.g. a missing document in the shared folder, but not know the actions that caused it, e.g. whether a convention was violated. Therefore, past history of actions is important in a shared workspace in order to reconstruct what actions have been done. Present actions and effects are necessary in order to be aware of the state of the documents. Awareness of future effects are necessary since one may see the current actions of others yet needs to learn their consequences. We argue that all three types of awareness information are necessary in order to overcome the cooperation difficulties raised by restricted feedback to aid users in establishing and maintaining conventions for groupware use.

\subsection{Providing cues for conventions}

The system should also provide cues about appropriate behavior in a shared workspace. Four levels of support are possible: signaling conventions by visualization; notification and provision of feedback about action, to support active learning of conventions; automatic ensurement of conventions, and enforcement of conventions, to avoid inappropriate user behavior.

The visualization of conventions through different workspace appearances is an implicit way of signaling conventions in a shared workspace. This can be achieved using appropriate interface metaphors such as rooms. Thus, the appearance of that workspace signals its type, and the conventions that apply to that type of workspace.

The metaphors applied by our groupware platform, LinkWorks, all stem from single user objects, e.g. cabinets, folders, and drawers. These are appropriate for the organization of a single user environment, but not for the organization of a shared environment. The semantic of these metaphors is applied to govern and control how containers are nested. They have no implication for their use as shared workspaces, nor do they change their behavior when they are shared. Often users were not aware whether the workspace they are using is shared or not.

Notification and the provision of feedback can support users in learning what behavior is appropriate. Notification can be used as reinforcement when a convention is correctly followed, and it can also promote active learning when the convention is broken. This technique increases the awareness of the users of 
the consequences of their actions, e.g. that moving an object out of a shared workspace makes that object inaccessible for other members of the shared workspace.

The automatic ensurement of conventions in our case means that an alias of a document is automatically created when it is dragged out of the folder. However, this automatic ensurement of the convention has the drawback that the user will not become aware of his/her actions, since the user does not actively perform the action. Thus, active learning does not occur.

The enforcement of conventions can hinder inappropriate behavior of users in a shared workspace. However, the enforcement of conventions can be too rigid of a solution since it may hinder cooperation. Enforcement would imply that users are not allowed to remove a document out of the shared folder, but that could prevent users from storing and representing documents in different folders when that is necessary for a specific task.

This discussion illustrates that an easy and straightforward solution for the support of group conventions in shared workspaces does not exist. The choice of the support level depends on the particular convention, its importance for the functioning of the group, and the groupware experience. For example, an essential convention in an inexperienced group can be supported by a combination of notifications and the provision of feedback to support the active learning and collection of experiences together with enforcements to warn users from unintended but harmful actions. In a more experienced group, the automatic ensurement of conventions becomes relevant combined with sporadic notification to refresh the users' awareness about the conventions. This multi-level support for conventions requires a very flexible approach that can adapt to the level of experience of a group (see Syri, 1997).

\subsection{Supporting individual conventions}

When individual conventions may be difficult for users to give up, a technical solution is to enable users to retain them. Not only does this offer the large advantage that users may keep conventions that might logically relate to their task, but also when users must assume other roles, such as substitutes, they can manage other users' files in a shared workspace using their own familiar routines.

In our case, different views of the shared documents were required by members of the writing office and
Unit 57 members. The writing office prefers the order view, which requires a two level structure of the documents. The Unit 57 members prefer the workprocess view, which requires that documents are further organized into folders where each folder represents a distinct process. But the writing office has no knowledge about the structure, so they cannot directly browse. The provision of a search facility is also not acceptable since a document is remembered by its location, not by its name.

The provision of different views is possible by the creation of additional aliases. By placing the alias of a document in a task specific folder, the unit members can organize documents in their individual views. However, subsequent changes to the document are still accessible for the writing office. Thus, the alias provides a translation between the different views.

We must now consider some problems that are raised when users have different views on shared information. Although a document is shared within a group, everybody has a different view to the document without a common reference, or group view. For example, a member of the Unit and a typist may refer to the same document differently, because they see the document from different views: different folders or workspaces. We must provide functions that map the individual views to a common group view. For a common reference, we must consider the identity and locality of shared objects in the individual views.

Object identity: users must be able to confirm whether two objects they are referring to are identical. This can be achieved either by a unique object share that is accessible by the users, or by a function that tells the users whether the object they both have currently selected are the same.

Object locality: to become aware of the structuring preferences of other users, it should be possible to get information about the locality of a shared object on another users' desk. This will allow a better understanding of other users who they collaborate with. However, a user might not want to publish certain information, e.g. if a user placed a shared document in the waste basket of his/her electronic desktop. Thus, it must be possible to assign appropriate access rights.

Thus, users can retain their own information structure while collaborating in the shared workspace. A common group view is available which is independent of the number, and configuration, of individual user views. Individual views are cognitively 
easier, especially when: 1) group membership is dynamic, i.e. new members can always adapt a file structure to their own needs, 2) the information becomes vast, and 3) one is a substitute for another member: they can simply access their own information structure to manage another user's workspace.

\section{CONCLUSIONS AND OUTLOOK}

In this paper we have presented our experience with the development of a user work group with PoLITeam. We discovered that the users need conventions for using the groupware as they gained experience with the system functionality and their electronic work process. We presented their user requirements for conventions and then illustrated a typical work scenario with PoliTeam, where following the conventions was critical. A technical solution was proposed for supporting group conventions, and for retaining some individual conventions using the groupware.

One might ask whether supporting individual conventions would lead users to think that the groupware system should be viewed as a single user, rather than group system, as Orlikowski discovered was prevalent thinking with one user group (Orlikowski, 1992). However, we argue that this would not be the case. First of all, other group conventions are required with the system, such as those for shared editing. Secondly, the emphasis on establishing conventions, both group and individual, using workshops, helps to reinforce a perspective that it is a group system where actions from each user produce consequences for others. And third, along with individual information structures, a reference view is available that represents a common view for the group, which users can refer to.

\section{ACKNOWLEDGMENTS}

We give special thanks to Alex Bordetsky, Ludwin Fuchs, Peter Hoschka, Markus Sohlenkamp, Anja Syri, and Volker Wulf for their valuable comments.

\section{REFERENCES}

Bales, G. (1950). Interaction Process Analysis. Chicago: University of Chicago Press.

Bennis, W. G. and Shepard, H. A. (1956). A theory of group development. Human Relations 9 (4), 415-437.
Bion, W. R. (1959). Experiences in Groups. London: Tavistock.

Dourish and Bellotti, V. (1992). Awareness and coordination in shared workspaces. In Proceedings of CSCW '92, Oct. 31-Nov. 4, Toronto, 107-114.

Hughes, J. A., Randall, D., and Shapiro, D. (1992). Faltering from ethnography to design. Proceedings of CSCW'92, Toronto, ACM Press, 115-122.

Klöckner, K., Mambrey, P., Sohlenkamp, M., Prinz, W., Fuchs, L., Kolvenbach, S., Pankoke-Babatz, U., Syri, A. (1995). PoliTeam: Bridging the Gap between Bonn and Berlin for and with the Users. Proc. ECSCW'95, Stockholm, Kluwer Academic, 17-31.

Leland, M. D. P., Fish, R. S, Kraut, R. E. (1988). Collaborative document production using Quilt. Proceedings of $C S C W^{\prime} 88$, Portland, OR, ACM Press.

Mambrey, P. Mark, G. and Pankoke-Babatz (1996). Integrating User Advocacy into Participatory Design: the Designers' Perspective. Proc. of PDC '96, 251259.

Moeller, G. (1954). The CS-UCS interval in GSR conditioning. Journal of Exper. Psych., 48:162-66.

Moreland, R. L. and Levine, J. M. (1989). Newcomers and oldtimers in small groups. In P. Paulus (ed.) Psychology of Group Influence. Hillsdale, N.J.: Lawrence Erlbaum.

Neuwirth, C. M., Kaufer, D. S., Chandhok, R., and Morris, J. H. (1990). Issues in the Design of Computer Support for Co-authoring and Commenting, Proceedings of CSCW'90, Los Angeles, CA., Oct. 1990.

Orlikowski, W.J. (1992). Learning from Notes: Organizational Issues in Groupware Implementation, Proc. of CSCW '92, Toronto, ACM Press, 362-369.

Posner, I. R. and Baecker (1992). How people write together. Proc. of the Twenty-fifth Hawaii Inter'l Conference on System Sciences, 127-138.

Prinz, W. and Kolvenbach, S., (1996). Support for workflows in a ministerial environment, Proceedings of CSCW'96 Boston, ACM Press, 199-208.

Schein, E. H. (1985). Organizational Culture and Leadership. San Francisco: Jossey-Bass.

Syri, Anja (1997). Tailoring Cooperation Support through Mediators. To appear in Proceedings of ECSCW' 97, Lancaster, Kluwer Academic.

Tatar, D. G., Foster, G., and Bobrow, D. G. (1991). Design for conversation: lessons from Cognoter. Int. J. Man-Machine Studies, 34, 185-209. 